\title{
Características de carcaça e qualidade de carne em linhagens da raça Nelore
}

\author{
Carcass and meat quality traits in lineages of Nellore breed
}

\begin{abstract}
Marina de Nadai Bonin ${ }^{\mathrm{I}^{*}}$ José Bento Sterman Ferraz $^{\mathrm{I}}$ Joanir Pereira Eler ${ }^{\mathrm{I}}$ Saulo da Luz e Silva ${ }^{\mathrm{II}}$ Fernanda Marcondes de Rezende ${ }^{\mathrm{III}}$ Diego de Córdova Cucco ${ }^{\mathrm{IV}}$ Minos Esperandio Carvalhov $^{\mathrm{V}}$ Roulber Carvalho Gomes da Silva ${ }^{\mathrm{I}}$ Elisângela Chicaroni de Mattos Oliveira ${ }^{\mathrm{I}}$
\end{abstract}

RESUMO

O objetivo deste estudo foi avaliar diferenças entre linhagens da raça Nelore para características de carcaça e qualidade de carne. Foram avaliadas treze linhagens da raça Nelore para as características de peso de carcaça quente, área de olho de lombo, espessura de gordura subcutânea, marmoreio e força de cisalhamento aos 7,14 e 21 dias de maturação. Para isso, foram utilizadas informações fenotípicas de 516 animais da raça Nelore e estimadas as diferenças esperadas na progênie para comparação entre as linhagens. Dentre os genearcas estudados, Golias obteve os melhores valores das diferenças esperadas na progênie para peso de carcaça quente $(+1,20 \mathrm{~kg})$, área de olho de lombo $(+0,88 \mathrm{~cm})$, marmoreio $(+3,47$ un $)$ e força de cisalhamento média (-0,09kg) e Akasamu para espessura de gordura subcutânea (+0,05mm). As diferenças entre linhagens da raça Nelore encontradas neste estudo podem ser utilizadas na escolha de touros para melhoria genética de características de carcaça e carne em rebanhos de gado de corte brasileiros.

Palavras-chave: Bos primigenius indicus, espessura de gordura subcutânea, força de cisalhamento, genearcas da raça Nelore, marmoreio.

\section{ABSTRACT}

The aim of this study was to evaluate the differences between lineages of Nellore breed for carcass and meat quality traits. Thirteen lineages of Nellore breed were evaluated from data of carcass and meat quality of 516 animals for estimating the expected progeny difference for hot carcass weight, rib eye area, fat thickness, marbling and shear force values at 7, 14 and 21 days of ageing. The founder Golias reached the better values of expected progeny difference for hot carcass weight $(+1.20 \mathrm{~kg})$, rib eye area
$(+0.88 \mathrm{~cm})$, marbling $(+3.47 \mathrm{un})$ and average shear force $(-0,09 \mathrm{~kg})$, and Akasamu the better value for fat thickness $(+0.05 \mathrm{~mm})$. The differences between lineages of Nellore breed found in this study couldbe used to select sires for genetic improvement of carcass and meat quality traits in Brazilian beef cattle herds.

Key words: rib eye area, Bos primigenius indicus, fat thickness, shear force, Nellore founders, marbling.

\section{INTRODUÇÃO}

O germoplasma de bovinos foi introduzido no Brasil há cerca de 450 anos pelos colonizadores, com animais oriundos de Portugal e Espanha e pertencentes à espécie Bos primigenius taurus. No final do século XIX, foram importados da Índia animais Bos primigenius indicus, também conhecidos como zebuínos. Esses animais se adaptaram muito bem ao Brasil, em função da semelhança das condições ambientais locais com as de seu país de origem, caracterizados pelo clima predominantemente tropical (MAGNABOSCO et al., 1997).

As importações e acasalamento de animais zebuínos com o gado bovino nacional fizeram surgir um período de "azebuamento" progressivo de considerável parcela do rebanho brasileiro, no qual a participação da raça Nelore foi de extrema importância

\footnotetext{
'Departamento de Medicina Veterinária, Faculdade de Zootecnia e Engenharia de Alimentos (FZEA), Universidade Federal de São Paulo (USP), Av. Duque de Caxias Norte, 225, Campus da USP, 13635-900, Pirassununga, SP, Brasil. E-mail: marinabonin@ @otmail.com.*Autor para correspondência.

"Departamento de Zootecnia (ZAZ), FZEA, USP, Pirassununga, SP, Brasil.

IIInstituto de Genética e Bioquímica, Universidade Federal de Uberlândia (UFU), Patos de Minas, MG, Brasil.

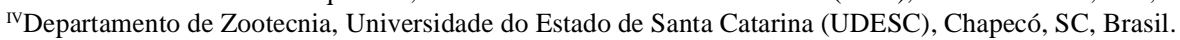

"Departamento de Zootecnia, Escola Superior de Agricultura “Luiz de Queiroz" (ESALQ), USP, Piracicaba, SP, Brasil. Recebido 08.08.13 Aprovado 07.02.14 Devolvido pelo autor 16.07.14 CR-2013-1073.R2
} 
(SANTIAGO, 1987). Existem indicações de que o número de zebuínos importados da Índia, até a década de 60, não ultrapasse 7.000 cabeças (MAGNABOSCO et al., 1997), no entanto, atualmente, cerca de $80 \%$ do rebanho bovino brasileiro possui genes oriundos desses animais (FERRAZ \& FELÍCIO, 2010).

As importações ocorridas na década de 60 foram especialmente marcadas pela vinda de animais excepcionais em características raciais e de desenvolvimento, o que trouxe apreciável melhoria para o rebanho brasileiro da raça Nelore. Alguns desses reprodutores tornaram-se conhecidos e granjearam fama como melhoradores de plantéis nos diferentes centros de criação de zebuínos da raça Nelore e passaram a ser classificados como genearcas ou formadores de linhagens dentro dessa raça (MAGNABOSCO et al., 1997).

Apesar do reduzido número de genearcas responsável pela formação do Nelore brasileiro, há evidências de diferentes perfis genéticos entre as linhagens. Diante disso, este estudo foi elaborado com o objetivo de avaliar diferenças entre linhagens da raça Nelore para características de carcaça e qualidade de carne.

\section{MATERIAL E MÉTODOS}

Foram utilizados 516 machos não castrados da raça Nelore, com idade média de 24 meses e abatidos com peso vivo médio de $550 \mathrm{~kg}$. Os abates foram distribuídos em seis lotes entre agosto e dezembro de 2006, todos realizados em frigorífico comercial, localizado na cidade de Promissão - SP, seguindo a legislação brasileira de inspeção sanitária e abate humanitário. Após o abate, as carcaças foram pesadas para obtenção do peso da carcaça quente (PCQ) e resfriadas a $2^{\circ} \mathrm{C} \pm 1^{\circ} \mathrm{C}$ por 24 horas, para mensuração do $\mathrm{pH}$ (pH24), da área de olho de lombo (AOL), espessura de gordura subcutânea (EGS) e marmoreio (MAR) no músculo Longissimus dorsi, entre a $12^{\mathrm{a}}$ e $13^{\mathrm{a}}$ costelas, utilizando, para esta última, o padrão USDA Quality Grade com escalas iniciando com Slight (400 unidades) a Moderately Abundant (900 unidades).

As análises de força de cisalhamento (FC) foram realizadas no Laboratório de Avaliação Animal e Qualidade de Carne da Faculdade de Zootecnia e Engenharia de Alimentos da Universidade de São Paulo (FZEA/USP), seguindo o método proposto pela AMSA (1995), a partir de amostras do músculo Longissimus dorsi, na altura da $12^{\mathrm{a}}, 11^{\mathrm{a}} \mathrm{e}$ e $10^{\mathrm{a}}$ costelas, maturadas por 7, 14 e 21 dias, respectivamente.

A avaliação genética dos animais foi realizada pelo Grupo de Melhoramento Animal e Biotecnologia da Faculdade de Zootecnia e Engenharia de Alimentos da Universidade de São Paulo (FZEA/USP), utilizando as estimativas das diferenças esperadas na progênie (DEP), realizadas por meio de modelos mistos sob modelo animal completo, pelo programa MTDFREML, que estimou os valores genéticos com base em informações de carcaça e carne de 516 animais e matriz de parentesco, composta por 3.982 animais.

As análises dos efeitos de cada genearca consideraram as DEP's para PCQ, AOL, EGS, MAR e FC em treze linhagens da raça Nelore, representadas pelos genearcas Taj Mahal (TAJM), Godhavari (GODH), Kurupathy (KURU), Golias (GOLI), Karvadi (KARV), Bima, Akasamu (AKAS), Padhu (PADH), Cacique (CACI), Nagpur (NAGP), Rastã (RAST), Checurupadu (CHEC) e Bazuá (BAZU). As DEP's foram consideradas como variáveis dependentes e a proporção de parentesco de cada genearca (Tabela 1) como covariável, em análises de regressão múltipla, numa estrutura de meioirmãos paternos. Nessa abordagem, foram estimados e testados os coeficientes de regressão linear das características em relação aos genearcas. Para tanto foi utilizado o procedimento PROC MIXED do pacote estatístico do Statistical Analysis System (SAS), versão 9.1.3 e o teste F, respectivamente.

\section{RESULTADOS E DISCUSSÃO}

As estatísticas descritivas das características avaliadas estão apresentadas na tabela 2 .

Foi encontrada alta variabilidade entre as linhagens da raça Nelore para produção de carcaças e carne com qualidade (Tabela 3). Alguns genearcas como Golias e Karvadi apresentaram potencial genético para produção de descendentes com maiores pesos de carcaça, músculos e também para carne mais macia. A linhagem Golias se destacou entre as demais linhagens avaliadas para deposição de gordura intramuscular e a linhagem Akasamu para gordura subcutânea.

As DEP's para as características de carcaça e carne deste estudo estão em conformidade com aquelas descritas na literatura, sendo comumente consideradas baixas quando comparadas às DEP's para características de crescimento (CREWS Jr, 2002). Já as acurácias foram baixas, o que também era esperado, visto o número relativamente pequeno de animais avaliados, do reduzido número de progênies e dos baixos coeficientes de parentesco de alguns genearcas com seus descendentes (Tabela 1). No entanto, há de se considerar que, mesmo com estas 
Tabela 1 - Número de descendentes e parentesco de cada genearca da raça Nelore para a composição dos genótipos dos animais avaliados para características de carcaça e qualidade de carne.

\begin{tabular}{|c|c|c|c|c|}
\hline \multirow{2}{*}{ Genearca } & \multirow{2}{*}{ № descendentes } & \multicolumn{3}{|c|}{----Coeficiente de Parentesco (\%)------- } \\
\hline & & Médio & Mínimo & Máximo \\
\hline Akasamu (AKAS) & 207 & 0,70 & 0,10 & 5,08 \\
\hline Bazuá (BAZU) & 340 & 0,81 & 0,01 & 4,10 \\
\hline Bima & 03 & 0,27 & 0,20 & 0,39 \\
\hline Cacique (CACI) & 41 & 0,08 & 0,20 & 1,95 \\
\hline Checurupadu (CHEC) & 492 & 2,92 & 0,10 & 16,46 \\
\hline Ghodavari (GHOD) & 194 & 0,26 & 0,05 & 2,34 \\
\hline Golias (GOLI) & 451 & 2,23 & 0,10 & 9,38 \\
\hline Karvadi (KARV) & 492 & 2,23 & 0,20 & 8,59 \\
\hline Kurupathy (KURU) & 194 & 0,23 & 0,07 & 2,34 \\
\hline Nagpur (NAGP) & 87 & 0,13 & 0,20 & 3,13 \\
\hline Padhu (PADH) & 231 & 0,33 & 0,10 & 3,13 \\
\hline Rastã (RAST) & 63 & 0,10 & 0,20 & 1,56 \\
\hline Taj Mahal (TAJM) & 225 & 0,18 & 0,01 & 1,61 \\
\hline
\end{tabular}

limitações, foi detectado um efeito genético positivo de linhagens da raça Nelore sobre características de carcaça e carne, indicando que outros estudos, com maior número de animais, devam ser conduzidos a fim de confirmar esses resultados.

Dentre as linhagens avaliadas, os genearcas GOLI e AKAS obtiveram os maiores e menores valores de DEP para PCQ, respectivamente, com uma diferença de $+1,67 \mathrm{~kg}$ entre eles (Tabela 3 ), revelando a superioridade de GOLI em produzir progênies com carcaças mais pesadas (Figura 1A). Os valores médios de DEP para PCQ encontrados neste estudo ficaram próximos aos relatados na literatura. SMITH et al. (2007), avaliando 467 animais da raça Brahman, encontraram valor médio de $+1,18 \mathrm{~kg}$, variando entre $-19,40$ a $+17,61 \mathrm{~kg}$, ELZO et al. (1998), em estudo envolvendo as raças Angus, Brahman e Brangus, encontraram valores médios de -0,4; +0,7 e +9,3kg, respectivamente, sendo que, para animais da raça Brahman, as variações foram de $-11,2 \mathrm{a}+14,4 \mathrm{~kg}$.

No presente estudo, as variações nos valores de DEP foram menores que as relatadas na literatura, variando de $-0,47 \mathrm{a}+1,20 \mathrm{~kg}$. Isso pode ser devido ao fato de que neste estudo os animais foram abatidos com peso vivo médio de $550 \mathrm{~kg}$, diminuindo a variação fenotípica para esta característica. No entanto, esta informação é importante e ressalta que, mesmo abatidos com pesos vivos semelhantes, as variações nos valores de DEP para AOL, EGS e MAR foram grandes, revelando a variabilidade entre linhagens da raça Nelore para deposição de músculos e gordura na carcaça.
O valor médio de DEP encontrado para AOL foi de $+0,09 \mathrm{~cm}^{2}$, variando entre $-0,58$ e $0,88 \mathrm{~cm}^{2}$ (Tabela 3), gerando uma diferença de $+1,46 \mathrm{~cm}^{2}$ entre o genearca com maior (GOLI) e menor (AKAS) valor de DEP. Segundo SILVA et al. (2003), a AOL apresenta alta correlação com o rendimento de cortes nobres da carcaça. Sendo assim, a linhagem GOLI se destacou entre as demais linhagens avaliadas quanto à capacidade de gerar descendentes com maior musculosidade na carcaça (Figura 1A).

SMITH et al. (2007), utilizando animais da raça Brahman, encontraram valores médios de DEP para AOL de $+0,19 \mathrm{~cm}^{2}$, variando de $-3,30 \mathrm{a}$ $+6,87 \mathrm{~cm}^{2}$. Quando comparamos os valores médios de DEP obtidos neste estudo aos estimados por SMITH et al. (2007), podemos concluir que ambas as raças, pertencentes a espécie Bos primigenius indicus, foram capazes de acrescentar, em média, $+0,10 \mathrm{~cm}^{2}$ na área de olho de lombo em seus descendentes, existindo, portanto, uma variabilidade entre touros destas raças para aumento da AOL. No entanto, ao efetuar comparações entre animais de diferentes raças, devemos considerar também que pertenciam a diferentes rebanhos e que foram gerados a partir da média fenotípica de cada população avaliada, podendo não ter o mesmo efeito quando consideradas em diferentes populações ou ambientes, como no caso do gado Nelore no Brasil.

Apenas três dos genearcas avaliados, AKAS, GOLI e PADH, apresentaram DEP positiva para EGS (Figura 1B). Diferentemente das características relacionadas à PCQ e AOL, a linhagem 
Tabela 2 - Estatísticas descritivas das características de idade ao abate (IABATE), peso de carcaça quente (PCQ), pH às 24 horas de resfriamento ( $\mathrm{pH} 24)$, área de olho de lombo (AOL), espessura de gordura subcutânea (EGS), marmoreio (MAR) e força de cisalhamento (FC) aos 7, 14 e 21 dias de maturação da carne de animais da raça Nelore.

\begin{tabular}{|c|c|c|c|c|c|c|}
\hline Característica & $\mathrm{N}$ & Média & Mín & Máx & DP & $\mathrm{CV}(\%)$ \\
\hline Idade ao abate (IABATE, dias) & 516 & 740,92 & 654,00 & 1021,00 & 46,28 & 6,25 \\
\hline Peso de carcaça quente (PCQ, kg) & 516 & 291,39 & 255,50 & 393,00 & 18,10 & 6,21 \\
\hline pH 24 horas (pH24) & 515 & 5,95 & 5,20 & 6,99 & 0,25 & 4,20 \\
\hline Área de olho de lombo (AOL, $\left.\mathrm{cm}^{2}\right)$ & 515 & 73,39 & 56,00 & 98,00 & 7,03 & 9,58 \\
\hline Espessura de gordura subcutânea (EGS, mm) & 514 & 4,42 & 1,00 & 15,00 & 2,00 & 45,25 \\
\hline Marmoreio (MAR, unidades) & 515 & 426,55 & 400,00 & 600,00 & 42,10 & 9,87 \\
\hline Força de cisalhamento aos 7 dias $(\mathrm{FC} 7, \mathrm{~kg})$ & 508 & 5,97 & 1,82 & 11,05 & 1,47 & 24,62 \\
\hline Força de cisalhamento aos 14 dias (FC14, kg) & 508 & 4,94 & 1,38 & 9,34 & 1,30 & 26,32 \\
\hline Força de cisalhamento aos 21 dias (FC21, kg) & 512 & 4,38 & 1,61 & 8,53 & 1,13 & 25,80 \\
\hline
\end{tabular}

$\mathrm{n}$ = número de observações; Mín = valor mínimo encontrado em cada característica; Máx = valor máximo encontrado em cada característica; $\mathrm{DP}=$ desvio padrão; $\mathrm{CV}=$ coeficiente de variação.

AKAS foi a que apresentou maiores valores de DEP para EGS.

Valores baixos ou próximos a zero de DEP para EGS são descritos na literatura. ELZO et al. (1998) encontraram valores de $+0,47 \mathrm{~mm}$ e SMITH et al. (2007) de $-0,01 \mathrm{~mm}$ para animais da raça Brahman. VAN VLECK et al. (2007), avaliando touros de diferentes raças, também encontraram valores próximos a zero, oscilando entre $-0,04 \mathrm{~mm}$ para touros Hereford e $+1,01 \mathrm{~mm}$ para touros Shorthorn. No presente estudo, esta variação foi de $-0,07$ a $+0,05 \mathrm{~mm}$, levando a uma diferença de $+0,12 \mathrm{~mm}$ entre os extremos, revelando a existência de variabilidade entre animais da raça Nelore para deposição de gordura subcutânea (Tabela 3, Figura 1B).

Para MAR, apenas cinco das treze linhagens avaliadas apresentaram DEP positiva (Tabela 3, Figura 1C), dentre elas, o genearca GOLI apresentou o maior valor que, comparado ao genearca com o menor valor (AKAS), gerou uma diferença de $+6,73$ unidades, revelando a existência de variabilidade e potencial genético de alguns genearcas da raça Nelore para deposição de gordura intramuscular na carcaça de suas progênies.

A gordura intramuscular tem sido associada a carnes de alta qualidade, pois contribui para melhoria da maciez, suculência e palatabilidade dos cortes (PLATTER et al., 2005). Sendo assim, animais com DEP positiva para MAR poderiam contribuir para melhoria da qualidade sensorial e valorização das carcaças de suas progênies.

GWARTNEY et al. (1996), utilizando progênies de touros da raça Angus com altos (-0,16 a +0,30un) e baixos $(-0,40$ a $+0,83$ un $)$ valores de DEP para marmoreio, para avaliar características de carcaça, encontraram que as progênies dos touros com altas DEP's para MAR tiveram também maiores valores de MAR em suas carcaças. DIKEMAN et al. (2005), avaliando os valores de DEP para marmoreio de animais das raças Simental, Shorthorn e Hereford, encontraram variações da ordem de $-0,22$ un a $+0,48$ un; de $-0,32$ a 0,00 un e de $-0,24$ a+0,40un, respectivamente. Neste estudo, as DEP's para MAR variaram de $-3,26 \mathrm{a}+3,47$ un (Tabela 3 ).

Estudos com animais Bos primigenius taurus têm demonstrado que, ao se selecionar para aumento do marmoreio nas carcaças, há a tendência de aumento da gordura subcutânea e também da gordura de aparas (MCPHEE et al., 2006). Neste estudo, no entanto, não necessariamente os maiores valores de DEP para MAR estiveram relacionados aos maiores valores de EGS (Tabela 3 ), sugerindo que esta tendência possa não ser verdadeira em animais da raça Nelore. Estes resultados estão de acordo com o relatado por GWARTNEY et al. (1996), que afirmam ser possível a utilização de DEP para MAR para seleção de touros, sem impactos na quantidade de gordura subcutânea na carcaça.

Para força de cisalhamento, todas as linhagens, com exceção de Bima e TAJM, apresentaram potencial para transmitir genes favoráveis à maciez da carne (Tabela 3, Figura 1D). Valores de força de cisalhamento são utilizados para avaliar a maciez da carne e indicam a medida da força exercida na amostra, em $\mathrm{kg}$, capaz de promover seu completo cisalhamento. Assim, quanto menor a força empregada para o rompimento das fibras, mais macia será a carne. Dessa maneira, valores de DEP negativos para esta característica 
Tabela 3 - Valores das diferenças esperadas na progênie (DEP) e suas respectivas acurácias (Ac), em \%, para as características peso de carcaça quente (PCQ), área de olho de lombo (AOL), espessura de gordura subcutânea (EGS), marmoreio (MAR) e força de cisalhamento (FC) aos 7, 14 e 21 dias de maturação, avaliadas em descendentes dos treze principais genearcas da raça Nelore no Brasil.

\begin{tabular}{lllllllllllllll}
\hline Gen & $\begin{array}{l}\text { PCQ } \\
(\mathrm{kg})\end{array}$ & $\begin{array}{l}\text { Ac } \\
(\%)\end{array}$ & $\begin{array}{l}\text { AOL } \\
\left(\mathrm{cm}^{2}\right)\end{array}$ & $\begin{array}{l}\text { Ac } \\
(\%)\end{array}$ & $\begin{array}{l}\text { EGS } \\
(\mathrm{mm})\end{array}$ & $\begin{array}{l}\text { Ac } \\
(\%)\end{array}$ & $\begin{array}{l}\text { MAR } \\
(\mathrm{un})\end{array}$ & $\begin{array}{l}\text { Ac } \\
(\%)\end{array}$ & $\begin{array}{l}\text { FC7 } \\
(\mathrm{kg})\end{array}$ & $\begin{array}{l}\text { Ac } \\
(\%)\end{array}$ & $\begin{array}{l}\text { FC14 } \\
(\mathrm{kg})\end{array}$ & $\begin{array}{l}\text { Ac } \\
(\%)\end{array}$ & $\begin{array}{l}\text { FC21 } \\
(\mathrm{kg})\end{array}$ & $\begin{array}{l}\text { Ac } \\
(\%)\end{array}$ \\
\hline Akas & $-0,47$ & 3,04 & $-0,58$ & 2,26 & 0,05 & 1,73 & $-3,26$ & 2,50 & 0,00 & 2,26 & $-0,06$ & 1,79 & 0,01 & 1,12 \\
Bazu & 0,77 & 1,46 & $-0,03$ & 1,02 & $-0,07$ & 0,00 & $-1,57$ & 1,15 & $-0,02$ & 0,78 & $-0,03$ & 0,27 & $-0,02$ & 1,12 \\
Bima & $-0,04$ & 0,01 & $-0,01$ & 0,00 & 0,00 & 0,00 & 0,03 & 0,01 & 0,00 & 0,00 & 0,00 & 0,27 & 0,00 & 0,00 \\
Caci & $-0,39$ & 0,27 & $-0,03$ & 0,19 & $-0,05$ & 0,00 & $-0,29$ & 0,21 & $-0,02$ & 0,78 & 0,00 & 0,27 & $-0,01$ & 0,00 \\
Chec & 0,29 & 0,06 & 0,00 & 0,00 & $-0,01$ & 0,00 & $-0,25$ & 0,03 & 0,00 & 0,00 & 0,00 & 0,27 & 0,00 & 0,00 \\
Godh & 0,73 & 0,58 & 0,23 & 0,40 & $-0,02$ & 0,00 & $-0,58$ & 0,45 & $-0,02$ & 0,78 & $-0,02$ & 0,27 & $-0,01$ & 1,12 \\
Goli & 1,20 & 3,08 & 0,88 & 2,47 & 0,01 & 1,73 & 3,47 & 2,73 & $-0,09$ & 2,26 & $-0,12$ & 3,30 & $-0,06$ & 3,05 \\
Karv & 0,96 & 3,17 & 0,44 & 2,26 & $-0,05$ & 1,73 & 0,24 & 2,53 & $-0,05$ & 2,26 & $-0,10$ & 1,79 & $-0,08$ & 1,12 \\
Kuru & 0,39 & 0,49 & 0,14 & 0,40 & $-0,02$ & 0,00 & $-0,71$ & 0,38 & $-0,01$ & 0,78 & $-0,01$ & 0,27 & $-0,01$ & 1,12 \\
Nagp & $-0,03$ & 0,36 & 0,16 & 0,19 & $-0,04$ & 0,00 & $-0,09$ & 0,31 & $-0,03$ & 0,78 & 0,00 & 0,27 & 0,01 & 1,12 \\
Padh & $-0,05$ & 0,58 & $-0,08$ & 0,40 & 0,01 & 0,00 & 0,61 & 0,45 & $-0,03$ & 0,78 & $-0,01$ & 0,27 & $-0,01$ & 1,12 \\
Rast & $-0,08$ & 0,23 & $-0,12$ & 0,19 & $-0,03$ & 0,00 & 0,36 & 0,16 & 0,00 & 0,78 & $-0,02$ & 0,27 & $-0,01$ & 0,00 \\
Tajm & 0,10 & 0,32 & 0,10 & 0,19 & $-0,04$ & 0,00 & $-0,74$ & 0,25 & 0,00 & 0,78 & 0,02 & 0,27 & 0,01 & 1,12 \\
\hline
\end{tabular}

Gen= genearcas, Akas $=$ Akasamu, Bazu=Bazuá, Caci=Cacique, Chec $=$ Checurupadhu, Godh=Godhavari, Goli $=$ Golias, Karv $=$ Karvadi, Kuru=Kurupathy, Nagp=Nagpur, Padh=Padhu, Rast $=$ Rastã, Tajm= Taj Mahal.

são desejáveis, sendo possível a seleção de animais com maior probabilidade de gerarem progênies com carnes mais macias. As linhagens GOLI e KARV destacaram-se por apresentarem melhores valores de DEP (mais negativos) em todos os períodos de maturação (Figura 1D).

PLATTER et al. (2005), pesquisando a propensão de consumidores norte-americanos em pagar mais por carnes de melhor qualidade, encontraram que cada aumento de um quilograma nos valores de força de cisalhamento estava associado a uma redução de US\$1,02 $\mathrm{kg}^{-1}$ no valor das carnes com menor maciez. Por isso, reprodutores que apresentam potencial genético para produção de progênies com carnes mais macias são desejáveis, e tendem a produzir filhos com carnes com maior valor agregado.

SMITH et al. (2007), avaliando touros da raça Brahman, encontraram valores DEP para força de cisalhamento, aos 7 dias de maturação, variando de $-0,36 \mathrm{a}+0,45 \mathrm{~kg}$ e entre $-0,16 \mathrm{e}+0,33 \mathrm{~kg}$ aos 14 dias de maturação, com médias de $+0,01$ e $0,00 \mathrm{~kg}$, respectivamente. ELZO et al. (1998), comparando animais das raças Angus, Brahman e Brangus, encontraram valores médios de DEP de $+1,02,+0,01$ e $-0,83 \mathrm{~kg}$, respectivamente. No presente estudo, as variações ficaram entre $-0,09$ e $0,00 \mathrm{~kg}$ para $\mathrm{FC} 7$, entre $-0,12$ e $+0,02 \mathrm{~kg}$ para FC14 e entre $-0,08$ e
$-0,01 \mathrm{~kg}$ para FC21, evidenciando variabilidade entre linhagens da raça Nelore para maciez, possibilitando a seleção de animais geneticamente superiores para produção de progênies com carnes mais macias, a partir de valores de força de cisalhamento.

\section{CONCLUSÃO}

Existe variabilidade genética entre linhagens da raça Nelore para características de carcaça e qualidade de carne. As linhagens Golias e Karvadi foram superiores para produção de progênies com maiores pesos de carcaça quente, área de olho de lombo, marmoreio e maciez e a linhagem Akasamu foi superior para deposição de gordura subcutânea. Essas diferenças podem ser utilizadas na escolha de touros para melhoria de características de carcaça, qualidade de carne em rebanhos de gado de corte.

\section{COMITÊ DE ÉTIICA E BIOSSEGURANÇA}

Tendo por base os princípios estabelecidos no Regimento Geral do Comitê de Ética em Experimentação Animal da FZEA/USP, informo que o projeto de pesquisa "Avaliação de características de desempenho e qualidade de carne em linhagens de touros representativos da raça Nelore, utilizado ultrassonografia, análise de imagens, NIRS”, coordenado pelo Prof. Dr. José B. S. Ferraz recebeu parecer favorável de um pesquisador que atua na área de experimentação animal. Deste modo, a Comissão de Érica em Experimentação Animal da FZEA considerou-o aprovado. 


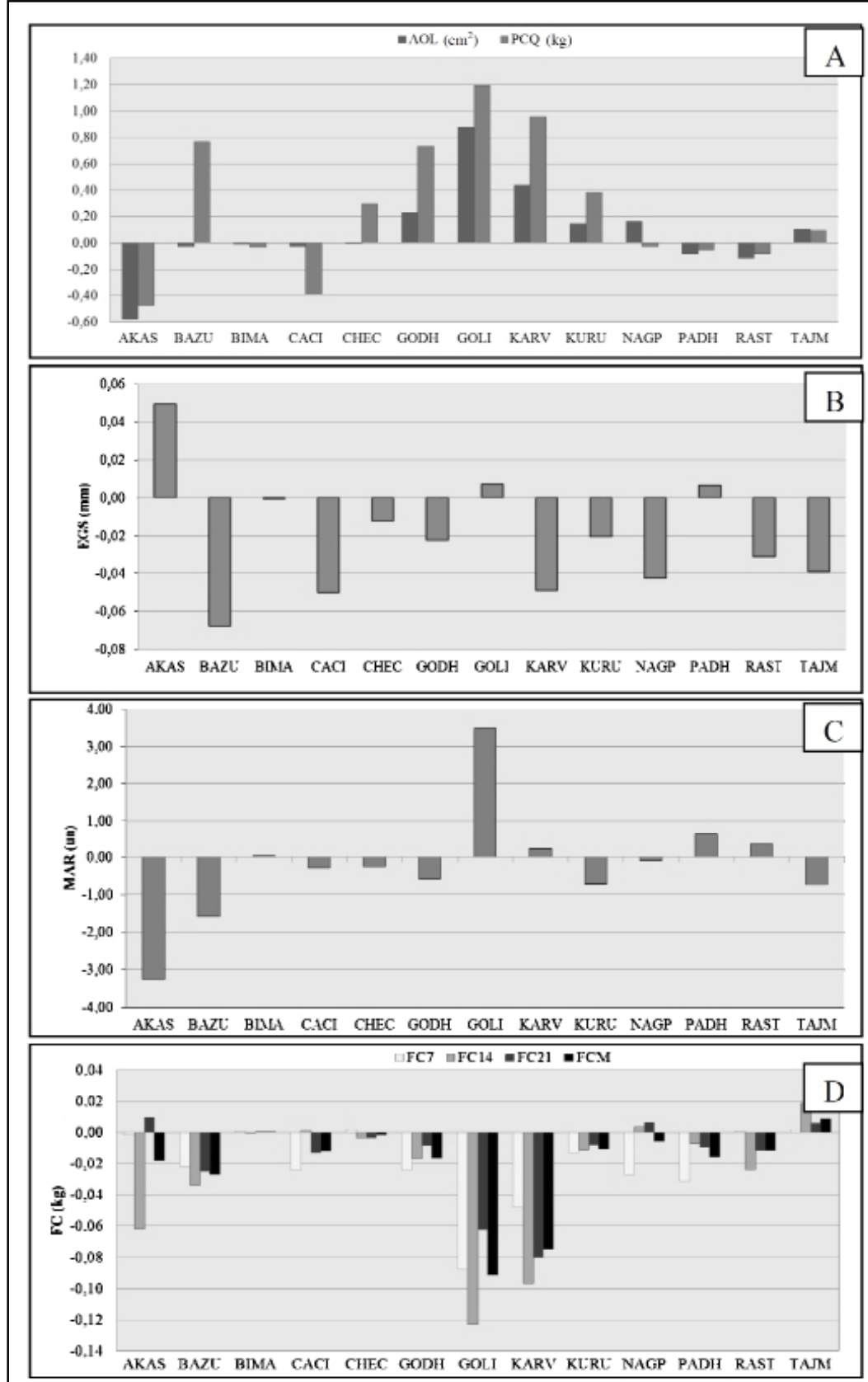

Figura 1 - Diferenças esperadas na progênie (DEP) para área de olho de lombo (AOL; A), peso de carcaça quente (PCQ; A), espessura de gordura subcutânea (EGS; B), marmoreio (MAR; C) e força de cisalhamento média (FCM; D), aos zero (FC0; D), sete (FC7; D) e quatorze (FC14; D) dias de maturação para os genearcas Akasamu (Akas), Bazuá (Bazu), Bima, Cacique (Caci), Checurupadhu (Chec), Godhavari (Godh), Golias (Goli), Karvadi (Karv), Kurupathy (Kuru), Nagpur (Nagp), Padhu (Padh), Rastã (Rast), Taj Mahal (Tajm).

\section{AGRADECIMENTOS}

Os autores agradecem a Fundação de Ampararo a Pesquisa de Ribeirão Preto (FUNPEC-RP) pelo apoio financeiro para realização desta pesquisa e à Agro-Pecuária CFM Ltda pelo fornecimento dos animais.

\section{REFERÊNCIAS}

AMERICAN MEAT SCIENCE ASSOCIATION (AMSA). Research guidelines for cookery, sensory evaluation, and instrumental tenderness measurements of meat. Chicago, 1995. 47p. 
CREWS Jr, D.H. The relationship between beef sire carcass EPD and progeny phenotype. Canadian Journal of Animal Science, v.82, p.503-506, 2002. Disponível em: <http:// dx.doi.org/10.4141\%2FA02-037>. Acesso em: 24 jul. 2012. doi: $10.4141 \% 2 \mathrm{FA} 02-037$.

DIKEMAN, M.E. et al. Phenotypic ranges and relationships among carcass and meat palatability traits for fourteen cattle breeds, and heritabilities and expected progeny differences for Warner-Bratzler shear force in three beef cattle breeds. Journal of Animal Science, v.83, p.2461-2467, 2005. Disponível em: <http:// www.journalofanimalscience.org/content/83/10/2461>. Acesso em: 16 jan. 2013.

ELZO, M.A. et al. Genetic variation and prediction of additive and nonadditive genetic effects for six carcass traits in an AngusBrahman multibreed herd. Journal of Animal Science, v.76, p.18101823, 1998. Disponível em: <http://www.journalofanimalscience. org/content/76/7/1810>. Acesso em: 30 jul. 2012.

FERRAZ, J.B.S.; FELÍCIO, P.E. Production systems - An example from Brazil. Meat Science, v.84, p.238-243, 2010. Disponível em: <http://dx.doi.org/10.1016\%2Fj.meatsci.2009.06.006>. Acesso em: 23 jan. 2013. doi: 10.1016\%2Fj.meatsci.2009.06.006.

GWARTNEY, B.L. et al. Use of expected progeny differences for marbling in beef: II. Carcass and palatability traits. Journal of Animal Science, v.74, p.1014-1022, 1996. Disponível em: <http:// www.journalofanimalscience.org/content/74/5/1014>. Acesso em: 12 jul. 2012.

MAGNABOSCO, C.U. et al. Catálogo de linhagens do germoplasma zebuíno: raça Nelore. Brasília: Embrapa-Cenargen, 1997. $52 \mathrm{p}$
MCPHEE, M.J. et al. Meta-analysis of factors affecting carcass characteristics of feedlot steers. Journal of Animal Science, v.8, p.3143-3154, 2006. Diponível em: <http://dx.doi. org/10.2527\%2Fjas.2006-175>. Acesso em: 15 jul. 2012. doi: $10.2527 \% 2$ Fjas.2006-175.

PLATTER, W.J. et al. Effects of marbling and shear force on consumers' willingness to pay for beef strip loin steaks. Journal of Animal Science, v.83, p.890-899, 2005. Disponível em: <http:// www.journalofanimalscience.org/content/83/4/890>. Acesso em: 05 ago. 2012

SANTIAGO, A.A. Gado Nelore: 100 anos de seleção. São Paulo: Editora dos Criadores. 1987. 592p.

SILVA, S.L. et al. Correlações entre características de carcaça avaliadas por ultra-som e pós-abate em novilhos Nelore, alimentados com altas proporções de concentrado. Revista Brasileira de Zootecnia, v.32, p.1236-1242, 2003.

SMITH, T. et al. Genetic parameters for growth and carcass traits of Brahman steers. Journal of Animal Science, v.85, p.1377-1384, 2007. Disponívelem: <http://dx.doi.org/10.2527\%2Fjas.2006-653>. Acesso em: 19 jul. 2012. doi: 10.2527\%2Fjas.2006-653.

VAN VLECK, L.D. et al. Across-breed adjustment factors for expected progeny differences for carcass traits. Journal of Animal Science, v.85, p.1369-1376, 2007. Disponível em: <http:// dx.doi.org/10.2527\%2Fjas.2006-658>. Acesso em: 15 jul. 2012. doi: $10.2527 \% 2$ Fjas.2006-658. 